\title{
Rapid recent radiation of S-RNase lineages in Witheringia solanacea (Solanaceae)
}

\author{
JL Stone and SE Pierce \\ Department of Biology, Colby College, Waterville, ME 04901, USA
}

\begin{abstract}
Strong frequency-dependent selection as found in the selfincompatibility loci of flowering plants maintains allelic lineages for extremely long time scales, such that allelic genealogies can shed insight into long-term demographic patterns of species. Effective mutation rate, as well as demographic change such as population bottlenecks, can influence genealogical structure. In addition, loss of functionality at the self-incompatibility locus is likely to affect radiation rates. Partial sequences for $21 \mathrm{~S}$-RNase alleles of the mid-elevation tropical species Witheringia solanacea were obtained in order to compare their substitution rates and genealogy with those of Witheringia maculata and two species in the closely related genus Physalis. Sequences for $W$. solanacea fell into the three clades within the Solanaceae already identified for the genus. Terminal branch lengths for $W$. solanacea, scaled to the total depth of its
\end{abstract}

phylogeny, were intermediate between the unusually short terminal branches of $W$. maculata and those of the two Physalis species. In contrast to the Physalis species, where interspecific $d_{N} / d_{S}$ for closely related alleles exceeded 1.0 to the same degree as did intraspecific $d_{N} / d_{S}$, in Witheringia only intraspecific comparisons showed an excess of nonsynonymous substitutions, suggesting postspeciation radiation of alleles. Alleles associated with lowered S-RNase production and self-compatibility showed extremely short terminal branches. In summary, it appears that rapid recent diversification of alleles characterizes the Witheringia lineages. In some cases, this rapid diversification can be attributed to relaxed constraints due to breakdown of selfincompatibility.

Heredity (2005) 94, 547-555. doi:10.1038/sj.hdy.6800657

Published online 16 March 2005

Keywords: coalescence; genealogy; self-compatibility; self-incompatibility; Solanaceae; S-RNase

\section{Introduction}

The power of negative frequency-dependent selection to maintain large numbers of alleles has long been recognized, with self-incompatibility (SI) alleles (Salleles) of flowering plants providing the classical example. Because only pollen bearing S-alleles distinct from those of the maternal plant can sire seeds, rare alleles are favored. Recent advances in molecular biology and in analytical techniques have promoted the reinvigoration of classical questions about equilibrium allele number, population structure, and mutation rates of S-alleles (reviewed by Clark, 1993), and have raised new questions as well. Allele genealogies can be scrutinized for departures from null expectation based on coalescent theory (Takahata, 1990; Vekemans and Slatkin, 1994). Population size should be revealed not only by number of alleles, but also by divergence time, with lineages from species with larger populations coalescing deeper in the tree. Differences in effective mutation rate (the effective rate of mutation to new Salleles) will also impact tree structure, with high effective mutation rates causing both high allele number and shallow coalescence (Uyenoyama, 1997). Much current work seeks to discern genealogical impacts of the

Correspondence: JL Stone, Department of Biology, Colby College, 5720 Mayflower Hill Dr., Waterville, ME 04901, USA.

E-mail: jstone@colby.edu

Received 18 May 2004; accepted 1 February 2005; published online 16 March 2005 interaction between effective mutation rate and demographic history.

The Solanaceae has been the focus of much of the work on S-allele genealogies from natural populations. Its SI response is characterized by an S-RNase that is expressed in the style of open flowers. The S-RNase degrades RNA of pollen that bears an allele not discernable as different from either of the two maternal alleles (McClure et al, 1990). Richman and Kohn have obtained partial S-RNase sequences from natural populations of five species (Richman et al, 1995; Richman and Kohn, 1999, 2000). Two of these species, Lycium andersonii and Solanum carolinense, each possess about a dozen trans-generic lineages and show unexpectedly long terminal branches when scaled to the total depth of the tree (Richman and Kohn, 2000). The other three species, from the closely related genera Physalis and Witheringia, together occupy only three lineages, representing a relatively shallow coalescence. Demographic effects may be responsible for the contrasts in tree shape between Physalis/Witheringia and other taxa (Richman and Kohn, 1999, 2000). A severe bottleneck along the lineage that led to Physalis and Witheringia may have drastically reduced the number of trans-generic lineages. Radiation of alleles subsequent to the bottleneck would result in relatively short terminal branches, in comparison with other genera that had not recently lost basal lineages.

In addition to demography, effective mutation rate and selective retention of alleles are also expected to influence tree shape. Uyenoyama (1997) modeled S-allele genealogies and found that divergence time among 
alleles is related linearly to effective population size. Effective mutation rate had an even more pronounced impact on divergence times, with low mutation rates increasing divergence times according to a power relationship. A third factor that potentially works with demography and effective mutation rate to influence tree-shape characteristics is the selective retention of alleles. Richman and Kohn (1999) suggest that more divergent alleles may be selectively maintained, perhaps because they are more likely to be recognized as nonself than are closely related alleles.

A fourth factor that could affect genealogical tree-shape characteristics, but that has not yet been considered in this light, is breakdown of functional SI of particular alleles. Breakdown of SI is an extremely common evolutionary trajectory in flowering plants (Stebbins, 1974), and can be caused either by mutations linked to the S-locus or by modifier genes (Stone, 2002). S-RNase alleles cosegregating with self-compatibility (SC), unlike their functional predecessors, are not subject to frequency-dependent selection. Lineages of such alleles will no longer persist for the extremely long time scales of functional alleles but, instead, may be expected to conform to neutral expectation (Vekemans and Slatkin, 1994). Therefore, in species where nonfunctional S-RNases have been able to invade, their diversification could be more rapid than those of functional S-RNases, resulting in relatively short terminal branch lengths. Counteracting the release from frequencydependent selection will be the extremely low effective population sizes of selfing lineages, which may deter diversification of these neutral alleles. Theoretical models have not yet treated the effects of polymorphism for SI on diversification rates of nonfunctional mating system alleles, but it is clear that effective mutation rate will itself be influenced by the breeding system.

It will not be simple to untangle the contribution of demography, effective mutation rates, selective retention, and mating system to tree-shape characteristics of Salleles. A potentially promising tactic is to focus intensively on particular genera with anomalous treeshape characteristics. An intriguing finding of earlier work was the report of unusually short terminal branch lengths in Witheringia maculata, a tropical cloud forest gap species (Richman and Kohn, 2000). According to the view that effective mutation rates are important, short branch lengths reveal rapid diversification due to an unusually high effective mutation rate. However, Richman and Kohn concluded that W. maculata displayed an extremely low diversification rate in comparison with Physalis, based on the low total number of alleles recovered and the inferred small population size. An examination of a second species in the genus will reveal whether short terminal branches are characteristic of the genus, due perhaps to similar effective mutation rates, or whether terminal branch lengths differ among congeners, perhaps due to differences in effective population size. Sequence comparisons among congeners can also provide information about timing of diversification with respect to speciation. If most diversification precedes speciation, the ratio of nonsynonymous to synonymous substitutions $\left(d_{\mathrm{N}} / d_{\mathrm{S}}\right)$ should be similar for inter- and intraspecific paired comparisons (Richman and Kohn, 1999). In contrast, if much diversification has occurred postspeciation, $d_{\mathrm{N}} / d_{\mathrm{S}}$ should be greater in intraspecific than in interspecific comparisons.
The objective of this research was to examine genealogical characteristics and nucleotide substitution rates for a second species of Witheringia, to see whether low diversification and evolutionary rates characterize postbottleneck recovery of this tropical genus. We selected Witheringia solanacea, which, like W. maculata, would have been restricted to canopy gap habitats until recent human habitat alterations have expanded suitable habitat to include roadsides and other disturbed areas. $W$. solanacea occupies slightly lower elevations than does W. maculata, and so in spite of its historically fugitive life history, could be expected to have a larger population size. Accessions of W. solanacea polymorphic for SI had previously been identified by Bohs (1999). Relictual SRNases from known self-compatible individuals were sequenced and included in an allele genealogy with SRNases from functional alleles. The following specific questions were addressed:

- Does W. solanacea have more S-alleles than W. maculata, as might be expected from its larger altitudinal range?

- Are terminal branch lengths in W. solanacea unusually short as found in W. maculata, suggesting similarly high effective mutation rates (to new $S$ alleles)?

- Do nonsynonymous substitution rates in Witheringia indicate a lack of postspeciation diversification as found in the closely related Physalis?

- Are nonfunctional S-RNases characterized by shorter terminal branches than are functional alleles?

\section{Materials and methods}

\section{Sampling from wild populations}

W. solanacea $\mathrm{L}^{\prime}$ Her. is a morphologically variable shrub occurring from southern Mexico to Central America and the Caribbean to South America (D'Arcy, 1973), reportedly at elevations from sea level to $2000 \mathrm{~m}$ (Standley, 1937). Individual plants were collected at a distance of at least $5 \mathrm{~m}$ from other individuals and transported as cuttings to the greenhouse at Colby College. See Table 1 for locations of collections. Five populations were sampled. Four of these populations consist of two pairs, one population each of relatively low and high elevations, in the southwest (Las Alturas and Las Cruces) and northwest (Monteverde and San Luis). Plants were identified as SC or SI based on observations of pollen tube growth following hand pollinations, and/or repeated autonomous production of progeny in a pollinator-free greenhouse. Most plants from these four populations rarely produce selfed seeds (Bohs, 1999; Stone, in preparation). A fifth population, Vara Blanca, occurs in north-central Costa Rica, and consists of a mixture of self-incompatible and self-compatible individuals (Bohs, 1999; Stone, in preparation). At all sites but Monteverde plants occurred in a compact area, and small sample sizes reflect either small population sizes or conservative sampling due to efforts to ensure that different ramets of the same genet were not inadvertently collected. Specimens from all populations have been verified as belonging to $W$. solanacea by taxonomist Lynn Bohs, and vouchers are deposited in the herbarium at the University of Costa Rica (Stone collection numbers 1500-1510). 
Table 1 Locations, habitats, sampling effort, and numbers of alleles found for populations of W. solanacea in Costa Rica

\begin{tabular}{llcc}
\hline Population & Location & Elevation $(m)$ & Habitat \\
\hline Las Alturas (LA) & $8^{\circ} 57^{\prime} \mathrm{N}, 82^{\circ} 51^{\prime} \mathrm{W}$ & 1600 & Forest plantation \\
Las Cruces (LC) & $8^{\circ} 47^{\prime} \mathrm{N}, 82^{\circ} 57^{\prime} \mathrm{W}$ & 1180 & Weedy garden \\
Monteverde (MV) & $10^{\circ} 18^{\prime} \mathrm{N}, 84^{\circ} 49^{\prime} \mathrm{W}$ & $1330-1540$ & Roadside \\
San Luis (SL) & $10^{\circ} 17^{\prime} \mathrm{N}, 84^{\circ} 48^{\prime} \mathrm{W}$ & 1100 & Roadside \\
Vara Blanca (VB) & $10^{\circ} 13^{\prime} \mathrm{N}, 84^{\circ} 10^{\prime} \mathrm{W}$ & 1360 & 3 \\
\hline
\end{tabular}

$\mathrm{N} 1=$ number of individuals sampled; N2 = number for which two alleles were found. Samples included one self-compatible individual at Monteverde and two self-compatible individuals at Vara Blanca.

\section{Sequencing of S-RNases}

Partial S-RNase sequences were obtained, essentially as described by Richman et al (1995). Styles collected 1-2 days before anthesis were processed immediately. Four to six styles were ground in liquid nitrogen, and Qiagen's RNeasy Plant Mini Kit was used to obtain stylar mRNA. Invitrogen's cDNA cycle kit was used to generate cDNA. Degenerate primers PR1 and PR3 (Richman et al, 1995), based on conserved regions C2 and C5 (Ioerger et al, 1991), were used to amplify approximately 490 base pairs of the SRNase gene. Thermal cycler conditions were modified for use with a thermocycler with a heated lid $\left(94^{\circ} \mathrm{C}\right.$, $\left.15 \mathrm{~s} ; 45^{\circ} \mathrm{C}, 60 \mathrm{~s} ; 72^{\circ} \mathrm{C}, 60 \mathrm{~s}\right)$. PCR products were cloned and then sequenced using an ABI Prism 310 capillary sequencer. Forward and reverse strands were compared using Sequencher. At least two separate inserts were sequenced for all but two alleles (S11 and S18). These two alleles each differed by several amino acids from other alleles (S7 and S4, respectively), which were each sequenced from four or more inserts, and which nestle within the large Physalis/ Witheringia clades.

\section{Allele number}

Individuals with functional gametophytic SI systems are expected to possess two different S-alleles, but sometimes PCR with degenerate primers is successful in recovering only one of the two. Individuals that have nonfunctional SI systems may also yield a single allele; these are more likely to be true homozygotes. For the following analyses, we treated alleles identically regardless of whether they were found in SI or SC individuals. We applied the Mantel statistic across all samples to see whether alleles were represented in equal frequency (Mantel, 1974; Richman et al, 1995). If alleles are represented in equal frequency, Paxman's (1963) formula can be used to estimate the total number of alleles in a population by

$$
n=N\left(1-(1-2 / N)^{r}\right)
$$

where $n$ is the number of alleles found, $r$ is the number of plants sampled, and $N$ is the estimated total number of alleles in the population. We estimated total allele number for the Monteverde population, and also for the combined Monteverde and San Luis populations, which are separated by $2.5 \mathrm{~km}$. We counted plants where only a single allele was amplified as representing half of an individual with respect to sampling effort.
Genealogy construction and analysis

The relationship among S-RNase sequences from $W$. solanacea and other S-RNases was examined using an interspecific phylogeny. To include relevant closely related species, we obtained sequences from GenBank for available Physalis cinerascens, Physalis crassifolia and $W$. maculata sequences, discarding from our analysis those sequences that span only regions $\mathrm{C} 2-\mathrm{C} 4$ and retaining those that span $\mathrm{C} 2-\mathrm{C} 5$. In order to provide context for the Physalis and Witheringia species, we included sequences from natural populations of $S$. carolinense and $L$. andersonii, which had been used in the analysis that revealed Witheringia to cluster within the three Physalis clades (Richman and Kohn, 2000). In addition, we chose to include a manageable number of sequences from species that had not appeared with Witheringia in any prior analysis. To select these sequences, we sampled at random throughout the Solanaceae by obtaining S-RNase sequences submitted to GenBank before the year 2000, for species where four or more sequences were deposited (Nicotiana alata S2, S3, S6, S7; Petunia hybrida S1, S3, SB1, SB2, and Solanum chacoense S2, S3, S11, S13). Solanaceous S-like RNases LeLE and LeLX (Kock et al, 1995) and Nalata NE (Dodds et al, 1996) were used as the outgroup. ClustalX was used to align inferred amino-acid sequences, using the BLOSUM series substitution matrices, a gap origination penalty of 10 and a gap extension penalty of 0.5 . The alignment was checked by eye to ensure that conserved motifs and cysteine residues were properly aligned (Ioerger et al, 1991). Small insertions/deletions combined with highly variable sequences created problems aligning the ultimate seven to nine amino acids; for analysis, the terminal 4-5 amino acids of the sequences were truncated following the conserved cysteine at amino-acid position 119/120 (position 160 of Ioerger et al, 1991). PHYLIP v3.573c (Felsenstein, 1995) was used to construct a neighbor-joining tree. Distances were first calculated using PROTDIST with the Dayhoff PAM 001 amino-acid substitution matrix. A bootstrap tree was based on 100 re-samplings.

Intraspecific phylogenies for both Witheringia species were built to allow analysis of scaled terminal branch lengths. For the intraspecific phylogenies, DNA sequences rather than predicted amino-acid sequences were used, due to the closer relationships among alleles. Kimura's two-parameter distance as implemented by PAUP (Swofford, 1998) was used to create the matrix for the neighbor-joining tree, with S. carolinense S9 used as the outgroup. We used KITSCH (Felsenstein, 1995) to calculate terminal branch lengths, after using the branch program of LINTREE (Takezaki et al, 1995) to ensure that 
the genealogy's sequences did not deviate significantly from clocklike behavior. Relative terminal branch lengths were assessed by

$$
R_{\mathrm{SD}}=S(1-1 / n) / D
$$

where $S$ is the sum of terminal branch lengths, $D$ the total depth of tree, and $n$ the number of alleles in the sample.

Significance of $R_{\mathrm{SD}}$ was evaluated by comparison with Uyenoyama's (1997) simulated expectations. Testing of the assumption of a molecular clock and calculation of $R_{\mathrm{SD}}$ was also carried out for S-RNase sequences of W. maculata, using DNA sequences from GenBank (accession numbers AF102065-AF102075).

\section{Nucleotide substitution rates}

Closely related pairs of alleles were chosen for analysis of synonymous and nonsynonymous substitution rates. For comparisons within each Witheringia species, we focused on sister alleles that occupied paired terminal branches on the species-level phylogeny. For W. maculata, there were four such pairs, and for $W$. solanacea, there were five. For interspecific comparisons, we chose sister alleles occupying paired terminal branches on the full phylogeny. There were five interspecific pairs. We wanted to focus on substitutions for functional S-alleles, so we excluded the known nonfunctional alleles S12 and S23, with their single (nonsynonymous) substitution, leaving four pairs of sister alleles for the within $W$. solanacea comparison. Number of synonymous and nonsynonymous substitutions, and number of potentially synonymous and nonsynonymous sites were calculated using SITES (Hey and Wakely, 1997). Synonymous and nonsynonymous substitution rates were calculated using the Nei and Gojobori (1986)method. Paired $t$-tests were used to compare nonsynonymous substitution rates within and outside of hypervariable regions (Ioerger et al, 1991) for both intraspecific and interspecific comparisons.

\section{Results}

\section{Allelic diversity and genealogy}

In our sample of 21 plants, we found a total of 21 different alleles (GenBank accession numbers AY454099AY454121). For our sample of 18 outcrossing plants, two alleles were amplified in each of 11 individuals, and one allele was amplified for each of the remaining seven individuals (Table 1). For the two self-compatible plants from Vara Blanca and one self-compatible plant found in Monteverde, only one allele each was amplified. Alleles sampled from the largest population, Monteverde, were found at San Luis, Las Cruces, and Las Alturas as well (Table 2). Alleles were represented in approximately equal frequency, as expected under frequency-dependent selection, and assessed using the Mantel statistic $\left(\chi^{2}=17.5, P>0.5\right)$. Because the hypothesis of equal frequencies was not rejected, we were able to use Paxman's (1963) formula to estimate the total number of alleles. For the Monteverde population only, $n=12$ alleles were recovered from $r=8$ individuals, and the estimated number of alleles in the population is 24. For Monteverde pooled with San Luis, $n=15$ alleles were recovered from $r=10$ individuals, and the estimated number of alleles in the combined populations is 30 . Extension of the analysis to include the entire area sampled yields $n=21$ alleles, $r=16$ individuals, and an estimated total of 31 alleles. If self-compatible plants are excluded, estimated allele numbers are 21 for Monteverde only, and 35 for Monteverde plus San Luis and for the entire range sampled.

S-RNase sequences for $W$. solanacea clustered in the three Physalis/Witheringia clades already identified by Richman and Kohn (1999, 2000; Figure 1). For convenience, Clade 1 in the figure can be arbitrarily divided into three subclades, the central one of which has strong bootstrap support. W. solanacea sequences were not distributed evenly across the three clades (Table 2). Nine of them occur in clade 3 , along with nine W. maculata and Physalis sequences (Figure 1). W. solanacea alleles also dominate in clade 2, where they represent three of the five alleles, and in subclade 1B. In contrast, only one $W$. solanacea sequence appears among the 17 sequences in clade 1A, which is dominated by Physalis species. Lineage sorting among Physalis and Witheringia indicates substantial diversification of alleles after the divergence of the two genera.

\section{Terminal branch lengths}

The ordinary least squares method of Takezaki et al (1995) failed to reject deviation from a molecular clock for S-RNase genealogies of both $W$. maculata $(Q=11.5$, $\mathrm{df}=10, P>0.1)$ and $W$. solanacea $(Q=20.2, \mathrm{df}=20$, $P>0.1)$. The scaled ratio of terminal branch length to total depth, $R_{\mathrm{SD}}$, of 1.81 for $W$. maculata did not differ significantly from the expected value of 1.40 as predicted by simulation (Uyenoyama, 1997). $R_{\mathrm{SD}}$ for $W$. solanacea was much larger, 2.73, similar to the values found for Physalis species, which show a marginally significant trend toward long terminal branches (Uyenoyama, 1997; Richman and Kohn, 2000). The two W. solanacea alleles

Table 2 Allele frequencies by clade

\begin{tabular}{llll}
\hline Clade & Alleles & Frequencies & Populations \\
\hline 1a & S19 & 1 & LA \\
1b & S1, S2, S8, S9 & $4,1,1,1$ & MV, MV, MV, MV \\
$1 \mathrm{c}$ & S12, S13, S14, S23 & $2,2,2,2$ & MV and SL, MV and LC, MV and LC, VB \\
2 & S7, S11, S21 & $1,1,1$ & MV, MV, LC \\
3 & S6, S22, S5, S4, S18 & $1,1,2,1,1$ & MV, VB, MV and SL, MV and LC, LA \\
3 & S3, S15 & 3,1 & LA and SL, SL \\
3 & S16, S20 & 1,1 & MV, LC \\
\hline
\end{tabular}

See Figure 1 for clade designations and Table 1 for population codes. Frequency is number of occurrences out of the 31 allele copies sequenced. 


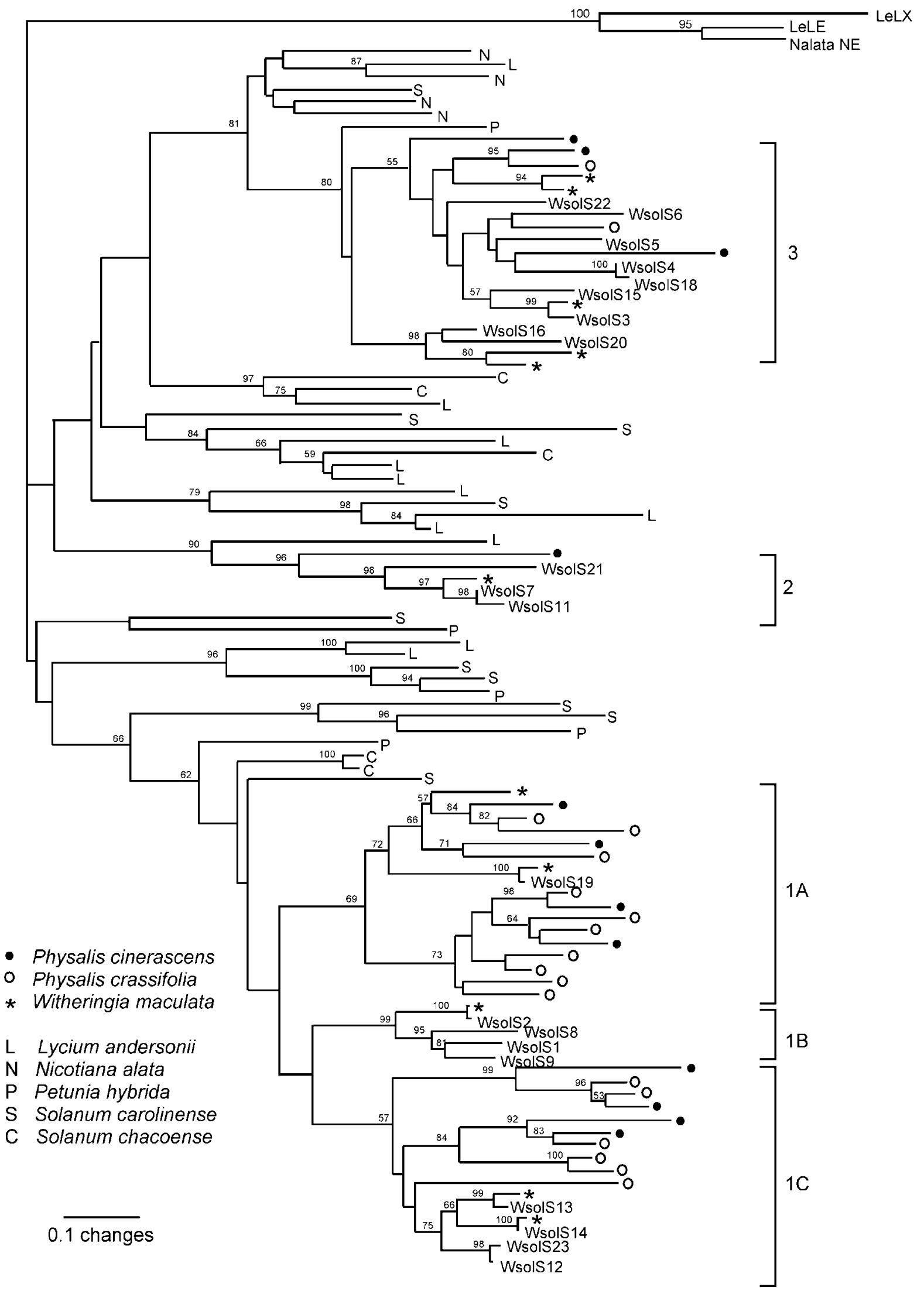

Figure 1 Neighbor-joining tree showing relationships among S-RNase amino-acid sequences spanning regions C2-C5 for W. solanacea, $W$. maculata, P. cinerascens, P. crassifolia, and other members of the Solanaceae (L. andersonii, N. alata S2, S3, S6, S7; P. hybrida S1, S3, SB1, SB2; S. carolinense; S. chacoense S2, S3, S11, S12, S13). Sequences for all species other than $W$. solanacea were obtained from GenBank. The outgroup consists of S-like RNases from Lycopersicon and Nicotiana. 
associated with SC, S12 and S23, have the shortest terminal branch lengths of any Wsol alleles (0.0014, as compared with an average of 0.059). Wsol branches S4, S7, and S14 that appear to be of zero length on the aminoacid phylogeny have an average terminal branch length of 0.014 on the species-specific phylogeny: longer than that of the SC alleles because of the length of the branch connecting them to the nearest intraspecific allele.

\section{Substitutions among closely related alleles}

Several W. solanacea S-RNase sequences were very similar to other sequences, both intra- and interspecifically (Figure 2). Wsol S12 and Wsol S23 differ for only a single replacement substitution at consensus amino-acid position 128 . This substitution was validated by independent sequences obtained on two different dates and for two different plants for each allele. For the case of S12, the sequence was found in one plant from Monteverde and another at lower elevation in San Luis. For the case of S23, the sequence was found for two different plants from Vara Blanca. Other pairs of similar sequences within $W$. solanacea differed by at least three nucleotides. Interspecific sister alleles and sister alleles for $W$. solanacea can be seen on the phylogeny in Figure 1. Two pairs of the sister alleles for $W$. maculata are less apparent on the full phylogeny: S7 and S8 occur amidst numerous Physalis sequences in Clade 1A, and S1 and S10 occur interdigitated with W. solanacea S13 and S14 in Clade $1 \mathrm{C}$.

The Witheringia S-RNase alleles in Clade 1C (Wsol S12, S13, S14, S23, and Wmac S1, S10) showed a nonconser- vative amino-acid substitution from aspartic acid to glycine in position 118 of conserved region C4 of Ioerger et al (1991). Allele S23 occurs in the highly selfing plants of Vara Blanca, which exhibit extremely low stylar RNase production (Stone, in preparation). The closely related allele S12 appears to be in homozygous state (based on lack of amplification of a second allele) in an autogamous plant from Monteverde. The alleles S13 and S14 have been found only in heterozygous state. It is not yet clear whether the mutation in $\mathrm{C} 4$ is itself responsible for low $\mathrm{S}$ RNase activity and SC, whether other mutations leading to SC have relaxed selection pressure on this replacement substitution, or whether it is a coincidence that SC appears in the lineage with this mutation.

\section{Synonymous and nonsynonymous substitution rates in Witheringia}

Synonymous substitution rates for closely related alleles in intraspecific comparisons are equal to those in interspecific comparisons (Table 3; Mann-Whitney $\left.U=26.5, n_{1}=8, n_{2}=5, P>0.10\right)$. In contrast, nonsynonymous substitution rates for closely related pairs of intraspecific alleles exceed those for interspecific allele pairs (Table 3; Mann-Whitney $U=40, n_{1}=8, n_{2}=5$, $P<0.01)$. As a result, the $d_{\mathrm{N}} / d_{\mathrm{S}}$ ratio differs between intra- and interspecific comparisons, with $d_{\mathrm{N}} / d_{\mathrm{S}}$ exceeding 1 for closely related alleles within species and about 0.5 for closely related alleles between species (Table 3). Nonsynonymous substitutions for intraspecific comparisons occur with higher frequency in regions previously designated as hypervariable than in the remainder of the

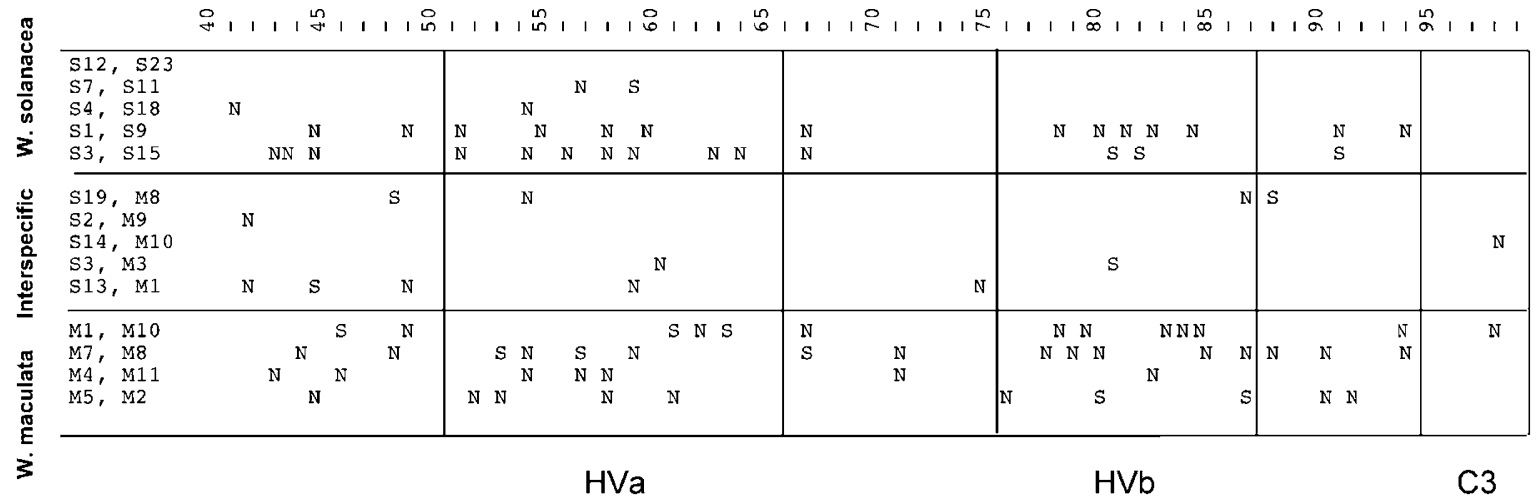

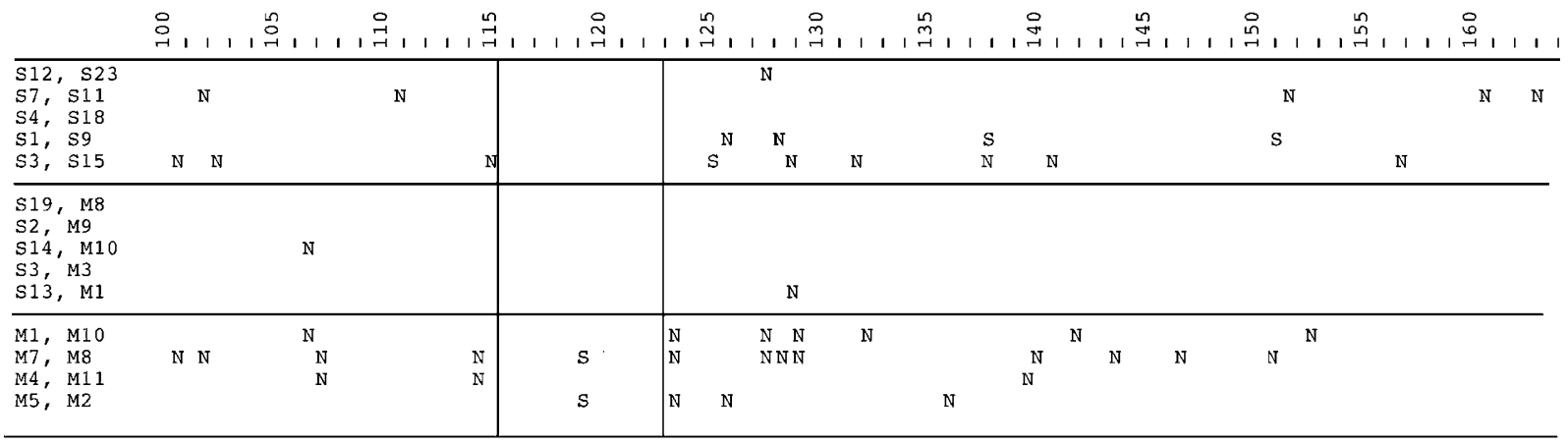

C4

Figure 2 Nonsynonymous (N) and synonymous (S) substitutions for closely related intra- and interspecific alleles of $W$. solanacea and W. maculata. Numbering system for amino-acid residues corresponds to Ioerger et al (1991). Previously identified hypervariable and conserved regions are identified by boxes. Multiple substitutions occurred for some amino-acid residues, and these are indicated with a single symbol. 
Table 3 Mean (standard error) of synonymous and nonsynonymous substitution rates for sister alleles

\begin{tabular}{lcccc}
\hline Allele pairs & $\mathrm{N}$ & $\mathrm{d}_{S}$ & $\mathrm{~d}_{N}$ & $\mathrm{~d}_{N} / \mathrm{d}_{S}$ \\
\hline W. solanacea & 4 & $0.045(0.020)$ & $0.052(0.021)$ & $1.245(0.377)$ \\
W. maculata & 4 & $0.066(0.012)$ & $0.078(0.019)$ & $1.247(0.242)$ \\
Interspecific & 5 & $0.036(0.008)$ & $0.011(0.005)^{*}$ & $0.385(0.078)^{*}$ \\
\hline
\end{tabular}

$N$ is number of sister alleles available for comparison. An asterisk indicates that the value differs from the others in the same column at $P<0.01$.

sequence (Figure 2; paired $t$-test, $P=0.005, \mathrm{df}=7$ ). For interspecific comparisons, in contrast, nonsynonymous substitutions occur at equal rates inside and outside of the hypervariable regions (paired $t$-test, $P>0.1, \mathrm{df}=5)$.

\section{Discussion}

\section{Ecological correlates of allele number}

W. solanacea has a similar life history to the previously investigated W. maculata: both historically occupied gaps in tropical forest canopies and therefore have had historically small, fugitive, populations. Estimated allele number of 24 for $W$. solanacea at Monteverde is larger than the 14.5 estimated for $W$. maculata at the same site. The larger altitudinal amplitude of $W$. solanacea, which is most commonly found between 1000 and $1500 \mathrm{~m}$, may be responsible for its large effective population size relative to $W$. maculata, which is restricted to elevations above $1500 \mathrm{~m}$. The effect of altitudinal range can be seen within our sample, with the inclusion of low elevation San Luis alleles resulting in an increase of estimated allele number from 24 to 30. Whether or not San Luis and Monteverde represent a panmictic population is an open question. Pollen is carried primarily by halictid bees (Stone, personal observation) and is unlikely to travel the $2.5 \mathrm{~km}$ separating the two areas. Gene flow by seed, however, is quite likely. W. solanacea fruits year-round; one of its primary dispersers at high elevations is Myadestes melanops (the Black-faced Solitaire; Murray, 1988), which migrates altitudinally into lowland forests when food is scarce in its montane breeding habitat (Stiles et al, 1989; Loiselle and Blake, 1991).

Although increased altitudinal range may explain differences in allele number between the two species of Witheringia, it remains puzzling how the effective population size of $W$. solanacea can be so much larger than that of several temperate fugitive species. For example, horsenettle (S. carolinense), being found over the entire eastern United States, has a much larger distribution than $W$. solanacea, but has a predicted allele number of only 11-18, with almost complete overlap of alleles found in two populations separated by $240 \mathrm{~km}$. Allele number for $W$. solanacea, in spite of its apparently weedy lifestyle, is intermediate between horsenettle and that found in temperate species such as P. crassifolia and $L$. andersonii, which are considered to display relatively large, stable population sizes (Richman and Kohn, 2000). It will be interesting to see whether high allele number characterizes tropical mid- to low-elevation species, where relatively stable climatic conditions may allow persistence of high allele number even for those species with fugitive life history strategies.
An issue in interpretation of allele number as obtained solely by molecular techniques is the question of whether different sequences necessarily correspond to different specificities, especially as here, when individuals from a broad geographic range are compared. Within a population, small $N_{\mathrm{e}}$ of specificities should eliminate multiple functionally equivalent variants (Takahata, 1990). Across populations, however, it is possible that slightly different amino-acid sequences could represent alternative forms of equivalent specificities. Hand crossing experiments are required to determine whether or not two closely related alleles are functionally equivalent. In the absence of compatibility data from crossing experiments, cooccurrence of closely related alleles within a population can be used to infer functional nonequivalence. For three of the four pairs of closely related SI alleles reported here, both sequences were found segregating within a single population. For one pair of closely related alleles (Wsol S4, Wsol S18), the two alleles were not found together in any population. Wsol S4 was found in Monteverde and $260 \mathrm{~km}$ away in Las Cruces, and Wsol S18 was found only at Las Alturas, about $23 \mathrm{~km}$ from Las Cruces. These alleles differ only by one synonymous and two nonsynonymous substitutions, and may represent a single functional allele. If the two alleles are functionally equivalent, allele diversification rate would be slightly lower than estimated here, although inferences about the excess of nonsynonymous substitutions in Witheringia would remain unchanged.

\section{Short terminal branch lengths are not a general characteristic of Witheringia}

Previous work has shown that whereas S-RNase alleles from Lycium and Solanum have extremely long terminal branches compared to neutral expectation, S-RNase alleles from Physalis have terminal branch lengths that are only marginally longer than predicted (Uyenoyama, 1997; Richman and Kohn, 2000). Explanations for long terminal branches include the presence of sheltered load reducing diversification rate (Uyenoyama, 1997, 2003) and selective retention of divergent alleles (Richman and Kohn, 2000). The S-RNase genealogy of W. maculata is unique in showing scaled terminal branch lengths that easily met neutral expectation (Uyenoyama, 1997; Richman and Kohn, 2000). An objective of this study was to see whether unusually short terminal branches would prove to be characteristic of the genus Witheringia, suggesting that the forces causing deviation from neutral expectation did not apply there. We found that scaled terminal branches for $W$. solanacea were not particularly short, and are similar to those found in Physalis. The shape of the S-RNase genealogy for W. maculata remains anomalous. 
Substitution rates indicate postspeciation radiation Intraspecific comparisons of closely related alleles for both Witheringia species show an excess of nonsynonymous substitutions, whereas interspecific comparisons among Witheringia species do not. This observation, together with the concentration of nonsynonymous substitutions in previously identified hypervariable regions thought to be associated with allelic recognition, suggests that selection has promoted diversification of new alleles postspeciation. In contrast, for $P$. cinerascens and $P$. crassifolia, interspecific and intraspecific $d_{N} / d_{S}$ ratios are indistinguishable, at about 1.6-1.8 (Richman and Kohn, 1999). The inference from these data is that the lineages that led to the two Physalis species examined had already radiated into the currently observed lineages prior to the speciation event that separated them. Thus, although both Witheringia and Physalis shared the same demographic bottleneck, speciation within the genera occurred at different times with respect to allele diversification, at least for the pairs of species considered. A higher rate of postspeciation allelic diversification for Witheringia is perhaps unsurprising, as cpDNA sequences suggest that $W$. solanacea is basal to Physalis (Olmstead et al, 1999). Still, the extent of lineage sorting between the genera is extensive, showing substantial radiation since their divergence; this radiation postdates speciation for these Witheringia but not for these Physalis species.

Breakdown of SI permits short-term allelic diversification The shortest terminal branches on the $W$. solanacea SRNase genealogy were the two that have been characterized as associated with SC, as is expected when variants within these allele classes are subject to neutral rather than inverse frequency-dependent selection (Vekemans and Slatkin, 1994). Classical theory dictates that an allele conferring SC should either be able to spread to fixation, if inbreeding depression can be purged, or should be rapidly lost, if not (eg, Charlesworth and Charlesworth, 1979; Lande and Schemske, 1985). Recent theory suggests, in contrast, that SI and SC alleles may stably coexist under certain conditions (Uyenoyama et al, 2001; Porcher and Lande, 2005). It is interesting that the lineage representing alleles S12 and S23 has persisted long enough to diversify, suggesting that parameter combinations required for a stable polymorphism may occur in nature. That the two SC alleles were not found cooccurring in any single population is hardly surprising due to their small effective population size, but even species-level polymorphism suggests a relatively long residence time. More functional studies will be necessary to assess the influence of loss of SI on diversification rates, and to evaluate the degree to which SC alleles can persist in predominantly SI populations. Breakdown of SI needs to be considered along with effects of demography and effective mutation rate on diversification of S-RNase lineages.

\section{Acknowledgements}

We thank Josh Kohn for helpful discussions, Brie Drummond, Jill Wentzell, and Chris Blomberg for assistance in the laboratory, Miruna Sasuclark for greenhouse support, and three anonymous reviewers for helpful comments. This work was supported by the Clare Boothe Luce Program of the Henry Luce Foundation and by funds from Colby College's Division of Natural Sciences.

\section{References}

Bohs L (1999). Insights into the Witheringia solanacea (Solanaceae) complex in Costa Rica. I. Breeding systems and crossing studies. Biotropica 32: 70-79.

Charlesworth D, Charlesworth B (1979). The evolution and breakdown of S-allele systems. Heredity 43: 41-55.

Clark AG (1993). Evolutionary inferences from molecular characterization of self-incompatibility alleles. In: Takahata N, Clark AG (eds) Mechanisms of Molecular Evolution. Sinauer: Sunderland, MA, pp 79-108.

D'Arcy WG (1973). Flora of Panama. Ann Miss Bot Gard 60: 573-580.

Dodds PM, Clarke AE, Newbigin E (1996). Molecular characterization of an S-like RNase of Nicotiana alata that is induced by phosphate starvation. Plant Mol Biol 31: 227-238.

Felsenstein J (1995). PHYLIP (Phylogeny Inference Package). Distributed by the author. Department of Genetics, University of Washington: Seattle.

Hey J, Wakely J (1997). A coalescent estimator of the population recombination rate. Genetics 145: 833-846.

Ioerger TR, Gohlke JR, Xu B, Kao T-h (1991). Primary structural features of the self-incompatibility protein in Solanaceae. Sex Plant Reprod 4: 81-87.

Kock M, Loffler A, Abel S, Glund K (1995). cDNA structure and regulatory properties of a family of starvation-induced ribonucleases from tomato. Plant Mol Biol 27: 477-485.

Lande R, Schemske DW (1985). The evolution of self-fertilization and inbreeding depression in plants I. Genetic models. Evolution 39: 24-40.

Loiselle BA, Blake JG (1991). Temporal variation in birds and fruits along an elevational gradient in Costa Rica. Ecology 72: 180-193.

Mantel N (1974). Approaches to a health research occupancy problem. Biometrics 30: 355-362.

McClure BA, Gray JE, Anderson MA, Clarke AE (1990). Self-incompatibility in Nicotiana alata involves degradation of pollen rRNA. Nature 347: 757-760.

Murray KG (1988). Avian seed dispersal of three neotropical gap-dependent plants. Ecol Monogr 58: 271-298.

Nei M, Gojobori T (1986). Simple methods for estimating the numbers of synonymous and nonsynonymous nucleotide substitutions. Mol Biol Evol 3: 418-426.

Olmstead RG, Sweere JA, Spangler RE, Bohs L, Palmer JD (1999). Phylogeny and provisional classification of the Solanaceae based on chloroplast DNA. In: Nee M, Symon DE, Jessup JP, Hawkes JG (eds) Solanaceae IV: Advances in Biology and Utilization. Royal Botanic Gardens: Kew, pp 111-137.

Paxman G (1963). The maximum likelihood estimation of the number of self-sterility alleles in a population. Genetics 48 : 1029-1032.

Porcher E, Lande R (2005). Loss of gametophytic self-incompatibility with evolution of inbreeding depression. Evolution 59: 46-60.

Richman AD, Kao T-H, Schaeffer SW, Uyenoyama MK (1995). S-allele sequence diversity in natural populations of Solanum carolinense (Horsenettle). Heredity 75: 405-415.

Richman AD, Kohn JR (1999). Self-incompatibility alleles from Physalis: implications for historical inference from balanced genetic polymorphisms. Proc Natl Acad Sci USA 96: 168-172.

Richman AD, Kohn JR (2000). Evolutionary genetics of selfincompatibility in the Solanaceae. Plant Mol Biol 42: 169-179.

Standley PC (1937). Flora of Costa Rica. Field Museum of Natural History, Botanical Series 18: Chicago, IL. 
Stebbins GL (1974). Flowering Plants: Evolution above the Species Level. Harvard: Cambridge, MA.

Stiles FG, Skutch AF, Gardner D (1989). A Guide to the Birds of Costa Rica. Cornell University Press: Ithaca, NY.

Stone JL (2002). Molecular mechanisms underlying the breakdown of gametophytic self-incompatibility. Quart Rev Biol 77: $17-32$.

Stone JL. Variation in stylar RNase production and selfincompatibility response within and among populations of Witheringia solanacea, unpublished data.

Swofford DL (1998). PAUP*. Phylogenetic Analysis using Parsimony (*and Other Methods), Version 4. Sinauer: Sunderland, MA.

Takahata N (1990). A simple genealogical structure of strongly balanced allelic lines and trans-species evolution of polymorphism. Proc Natl Acad Sci USA 87: 2419-2423.
Takezaki N, Rzetsky A, Nei M (1995). Phylogenetic test of the molecular clock and linearized trees. Mol Biol Evol 12: 823-833.

Uyenoyama MK (1997). Genealogical structure among alleles regulating self-incompatibility in natural populations of flowering plants. Genetics 147: 1389-1400.

Uyenoyama MK (2003). Genealogy-dependent variation in viability among self-incompatibility genotypes. Theor Popul Biol 63: 281-293.

Uyenoyama MK, Zhang Y, Newbigin E (2001). On the origin of self-incompatibility haplotypes: transition through self-compatible intermediates. Genetics 157: 1805-1817.

Vekemans X, Slatkin M (1994). Gene and allelic genealogies at a gametophytic self-incompatibility locus. Genetics 137: 1157-1165. 